\title{
Variant RON $\Delta 160$ of the RON receptor tyrosine kinase promotes the growth and invasion in vitro and in vivo in gastric cancer cell lines
}

Dong-Hui Zhou ${ }^{1 *}$, Chao Li ${ }^{1}$ and Li-Na Yang ${ }^{2}$

\begin{abstract}
Background: Recepteur d'origine nantais (RON) is a receptor tyrosine kinase whose overexpression has been observed in human gastric cancers. This study aimed to determine whether overexpression of the variant RON $\Delta 160$ could induce tumorigenicity of gastric cancer cells in vitro or in vivo, and whether its specific small molecule inhibitor (Compound I) could inhibit the effect of RON $\Delta 160$.

Methods: We constructed human gastric cancer cell line MGC-803 that was stably transfected with a recombinant plasmid expressing RON $\Delta 160$, and the effect of RON $\Delta 160$ overexpression and macrophage-stimulating protein (MSP) activation on proliferation, migration and invasion abilities of MGC-803 cells were evaluated. Tumor-bearing mice with gastric cancer cells were used to analyze the effects of RON $\Delta 160$ overexpression and Compound I on implanted tumor growth.
\end{abstract}

Results: In vitro, overexpression of RONA160 in MGC-803 cells resulted changes to their cell morphology, and promoted cell proliferation, migration and invasion. In addition, overexpression of RON $\Delta 160$ increased the proportion of cells in the $S$ phase. The effect of RON $\Delta 160$ was significantly enhanced by induction of MSP inducing $(p<0.05)$. In vivo, RON 160 promoted the growth of MGC-803 cells in nude mice, including increased tumor size and weight, and lower tumor incubation period. The Compound I inhibited the tumorigenic abilities of RON $\Delta 160(p<0.05)$.

Conclusions: The results indicate that overexpression of the variant RON $\Delta 160$ altered the phenotype and tumorigenicity of MGC-803 cells. Its specific small molecule inhibitor could inhibit the effect of RON $\Delta 160$. Therefore, the variant RON $\Delta 160$ may become a potential therapeutic target for gastric cancer.

Keywords: Gastric cancer, RON, RONA160, MSP, Tumorigenicity

\section{Background}

Gastric cancer is one of the most common malignant tumors, whose onset is difficult to diagnose. At the time of definite diagnosis, most patients are at the middle or late stage, and their response therapy and prognosis are poor. Thus, it is especially important to identify new molecular markers and therapeutic targets for gastric cancer. Studies have shown that the abnormal expression of RON, a member of the large RTK protein family, plays an important role in the incidence and development of tumors [1]. RON also plays an important role in the growth,

\footnotetext{
*Correspondence: zdh19838@163.com

'Department of Oncology, First Affiliated Hospital, Zhejiang University School of Medicine, Hangzhou 310003, China

Full list of author information is available at the end of the article
}

differentiation and proliferation of epithelial cells. RON is barely or not expressed in normal epithelial tissue, while in breast, colon, lung, thyroid, skin, bladder and pancreas tumors it is highly expressed and is usually accompanied by the generation of variants [2,3]. Currently, nine types of RON variants have been identified from primary carcinoma and cell lines, which are termed RON $\Delta 170$, RON $\Delta 165$, RON $\Delta 165 . e 11 p, \quad R O N \Delta 160, R^{\mathrm{E} O N / 6 i n}$, RON $\Delta 155, R O N \Delta \mathrm{p} 110, \mathrm{RON} \Delta 85$ and $\mathrm{RON} \Delta 55$, respectively [4-7]. These variants are generated by three main mechanisms: alternative splicing, protein truncation and alternative transcription of precursor RON mRNA [8]. $\mathrm{RON}$ is activated mainly in a ligand dependent manner. Currently, the sole known ligand of RON is macrophagestimulating protein (MSP). Maturate MSP is formed by 
the binding of the $53 \mathrm{kDa} \alpha$ chain with the $30 \mathrm{kDa} \beta$ chain through disulfide bonds. The binding of RON with the $\beta$ chain significantly increases RON's kinase activity and results in phosphorylation of the kinase domain [9]. RON contains functional domains that play important roles in ligand binding, protein maturation and bioactivity $[5,10]$. For example, deletion of the first immunoglobulin-plexintranscription (IPT) unit of the extracellular segment of $\beta$ chain produces variant RON $\Delta 160$, which is oncogenic and mediates many signals for tumor generation [11]. However, the effect of RON $\Delta 160$ on the incidence and development of gastric cancer has not been reported.

In this study, we explored the impact of RON $\Delta 160$ on the growth and invasion of MGC-803 cells by producing a derivative of MGC-803 that stably expresses high levels of RON $\Delta 160$. We also investigated the inhibitory action of a small molecule inhibitor of RON $\Delta 160$. This established the theoretical basis for exploring new therapeutic targets for the gastric cancer.

\section{Materials and methods Materials}

Human gastric cancer line MGC-803 was obtained from the State Key Laboratory for Diagnosis and Treatment of Infectious Diseases, The First Affiliated Hospital, Zhejiang University School of Medicine. PrimeScript ${ }^{\mathrm{m}}$ RT reagent kit and SYBR Premix Ex Taq ${ }^{\text {Tm }}$ reagent kits were purchased from Takara Biotechnology (Dalian Co., Ltd, China). Lipofectamine ${ }^{\mathrm{rm}} 2000$ and the cell cycle reagent kit were purchased from Invitrogen (Life Technologies, USA). Hygromycin B and c-Met/RON small molecular inhibitor (Compound I) were obtained from Calbiochem. Recombinant human MSP was purchased from R\&D systems. Cell counting kit 8 (CCK-8) was purchased from Dojindo Molecular Technologies (Rockville, USA). Antibodies against RON ( $\beta$-chain) were purchased from Santa Cruz Biotechnology (USA). The recombinant plasmid pcDNA3.1-RON $\triangle 160$ and blank plasmid pcDNA3.1 were obtained from Sangon Biotech Co., Ltd (China). Female Balb/c athymic nude mice $(\mathrm{Nu} / \mathrm{Nu})$ aged 6-weeks were obtained from the B\&K Universal Group and were kept in temperaturecontrolled rooms with a 12-h alternating light-dark cycle.

\section{Cell cultures and transfection}

MGC-803 cells were cultured in RMPI-1640 medium with $10 \%$ fetal calf serum (FCS). One day before transfection, cells were plated at a density of $60 \%-70 \%$ confluence. Transfection was made in the following groups: MGC-803 cells transfected pcDNA3.1-RON $\Delta 160$ (RON $\Delta 160$ group), MGC-803 cells transfected with blank plasmid pcDNA3.1 (empty vector control group), and non-transfected MGC803 cells (MGC-803 group). Transfection was conducted in accordance with the instruction of Lipofectamine ${ }^{\mathrm{Tw}} 2000$ reagent. After 24 hours, the cells were digested and diluted (1:10) with fresh culture media. After a further 24 hours $200 \mathrm{mg} / \mathrm{L}$ of Hygromycin B was added to select positive clones, which were obtained after about 2 weeks. Positive clones were expanded in culture medium with 200 mg/L Hygromycin B. Ultimately, MGC-803 cells stably expressing pcDNA3.1-RON $\triangle 160$ and blank plasmid pcDNA3.1 were obtained after about 8 weeks.

\section{Western blotting}

Proteins from each group were separated through $8 \%$ SDS-PAGE and then transferred to polyvinylidene difluoride (PVDF) membranes. The membranes were blocked with $5 \%$ nonfat milk for $2 \mathrm{~h}$ at room temperature and incubated with specific antibodies overnight at $4^{\circ} \mathrm{C}$. After thorough washing, the membranes were incubated with HRP-conjugated secondary antibody. Visualization of the reaction was conducted with enhanced chemiluminescence (ECL) reagents and analyzed by VersaDoc Imaging system (Bio-Rad).

\section{Quantitative real-time RT-PCR}

According to the manufacturer's instructions, the PCR reaction was carried out with the following primers: RON $\Delta 160$ forward: 5 ' -CTTGGCTGAGGTCAAGGAT GTG-3'; RON $\Delta 160$ reverse: 5'-CGATTCTGGGCCT GGTCTATG-3'; $\beta$-actin forward: 5'-CCAACCGCGAG AAGATGA-3'; $\beta$-actin reverse: 5'-CCAGAGGCGTAC AGGGATAG-3'. The amplification profile comprised precycling at $95^{\circ} \mathrm{C}$ for $20 \mathrm{~s}$; followed by 40 cycles of denaturation at $95^{\circ} \mathrm{C}$ for $15 \mathrm{~s}$, annealing at $60^{\circ} \mathrm{C}$ for $60 \mathrm{~s}$ and extension at $72^{\circ} \mathrm{C}$ for $40 \mathrm{~s}$.

\section{CCK-8 assay}

Cells were seeded into 96-well plates at a concentration of $2 \times 10^{3}$ cells/well, which were cultured in RPMI 1640 medium with $1 \%$ FCS. A RON $\Delta 160+$ MSP group of cells was created, to which were added MSP of final concentration 2nM. After seeding for 24-168 hours, the proportion of live cells was detected consecutively using CCK- 8 reagent. Finally, the absorbance (OD) value was measured with a microplate reader at wavelength of $450 \mathrm{~nm}$. The experiment was carried out in triplicate.

\section{Transwell invasion assay}

The cells were seeded at a density of $5 \times 10^{4}$ cells/chamber into the Transwell upper chamber $(8.0 \mu \mathrm{m}$ size micropore filtering membrane). The lower chamber was filled with $600 \mu \mathrm{l}$ of 10\% FCS containing RPMI 1640 culture media. After $24 \mathrm{~h}$ of incubation, the cells were fixed for 30 minutes with methanol. After washing with PBS, the cells that penetrated to the lower surface of the filter were stained with $0.1 \%$ crystal violet solution and counted under the microscope at a magnification $\times 400$ field. 


\section{Wound healing assay}

Cells from each group were seeded into 6-well plates at concentration of $5 \times 10^{5}$ cells/well, and cultured in $1 \%$ FCS-containing RPMI 1640 culture media. At 95\% confluence, the wounds were created with $200 \mu$ micropipette tip. MSP at a final concentration of $2 \mathrm{nM}$ was added into the RON $\triangle 160+$ MSP group for further stimulation. The cells were photographed at 0 hour and 48 hours after wounding, and the percentages of cell migration distance in 48 hours compared with the blank scratching distance at 0 hour were calculated.

\section{Cell cycle assay}

After digestion with trypsin, $2 \times 10^{5}-1 \times 10^{6}$ cells were collected, washed with PBS, fixed in pre-cooled $70 \%$ ethanol overnight at $-20^{\circ} \mathrm{C}$., The ethanol was removed after centrifugation, the cells were hydrated with PBS for 15 minutes, before $50 \mu \mathrm{l}$ RNase A was added and the cells incubated in a $37^{\circ} \mathrm{C}$ water bath for 30 minutes. Finally, $200 \mu \mathrm{l}$ of propidium iodide was added to cells for 30 minutes at $4^{\circ} \mathrm{C}$ with light-shielding to stain the cells. The cells were analyzed by flow cytometry. (FACScan, Calif., USA).

\section{In vivo tumor growth and treatment}

The effect of RON $\Delta 160$ on the tumorigenic ability of MGC-803 cells was determined by analyzing the subcutaneous tumor formation ability in 6-week-old BALB/c athymic female nude mice. The backs of the nude mice were subcutaneously planted with cells and the experiment was conducted in groups as follows: CP-I group was subcutaneously planted with MGC-803 cells stably transfected with RON $\Delta 160$, and from the second day they were intraperitoneally injected with the small molecule inhibitor c-Met/RON (Compound I) at dosage of $100 \mathrm{mg} / \mathrm{kg}$ for 4 weeks, twice every week; the RON $\Delta 160$ group was subcutaneously planted with MGC-803 cells stably transfected with pcDNA3.1-RON $\Delta 160$; the MGC-803 group was subcutaneously planted with MGC-803 cells; and control group was subcutaneously planted with MGC-803 cells transfected with the blank plasmid pcDNA3.1. The latter three groups were injected with normal saline using the same protocol the same as that for the CP-I group. The growth of subcutaneous-transplanted tumors was observed daily. After the formation of tumors, the tumor volume was measured every 3 days, and the incubation period of the tumor was recorded, i.e. the time from transplantation of tumor cells till the day when the tumor reached a volume of $500 \mathrm{~mm}^{3}$. After the tumor cells had been implanted for 40 days, the nude mice were killed by cervical disruption, the tumors were peeled off, and their volumes and weights were measured. All experiments involving mice were carried out using the recommendations of the Guide for the Care and Use of Laboratory Animals of the National Institutes of Health and were approved by the Zhejiang University Animal Care and Use Committee.

\section{Statistical analysis}

Data were summarized as mean \pm standard error $(\bar{X} \pm S)$. Statistical significance was determined by Student's $t$ test or by ANOVA, using SPSS 19.0 software. A
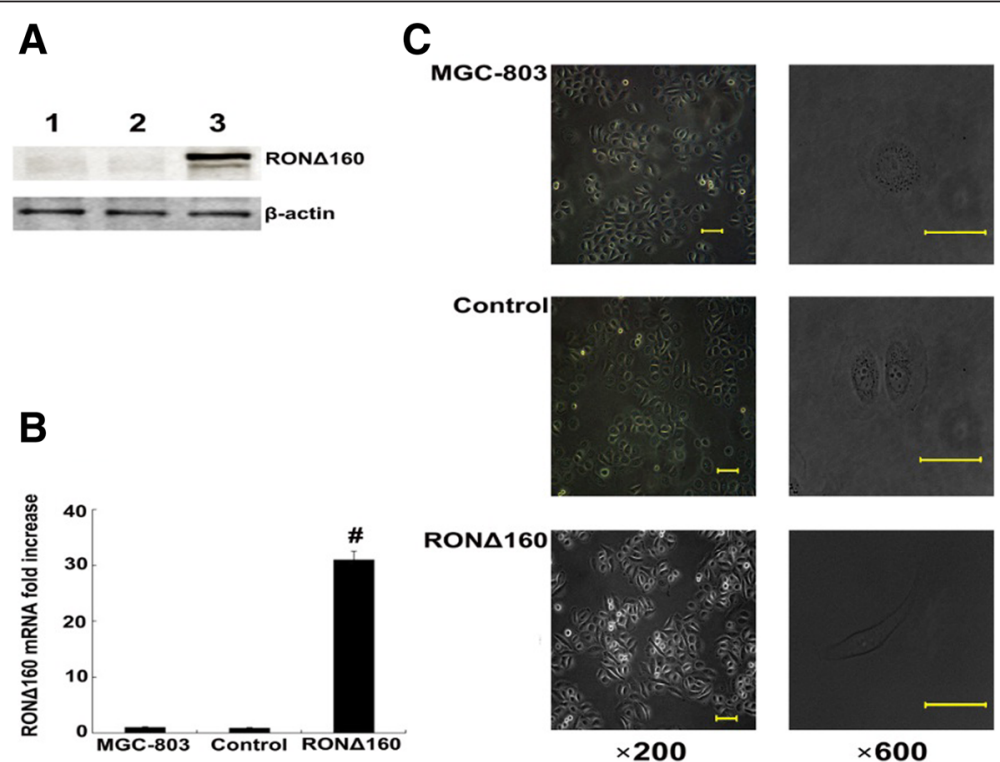

$\times 200$

$\times 600$

Figure 1 RON $\Delta 160$ is stably transfected into MGC-803 cell line and induces a change in morphology. A) Western blotting results: Lane 1, MGC-803 Group; Lane 2, empty vector control (control); Lane 3, RONA160 Group. B) Real-time RT-PCR result: $\beta$-actin mRNA was used as an internal control. \#: $p<0.01$. C) RON 160 induces a change in morphology from previous flat circular shape or polygon, to a spindle. 


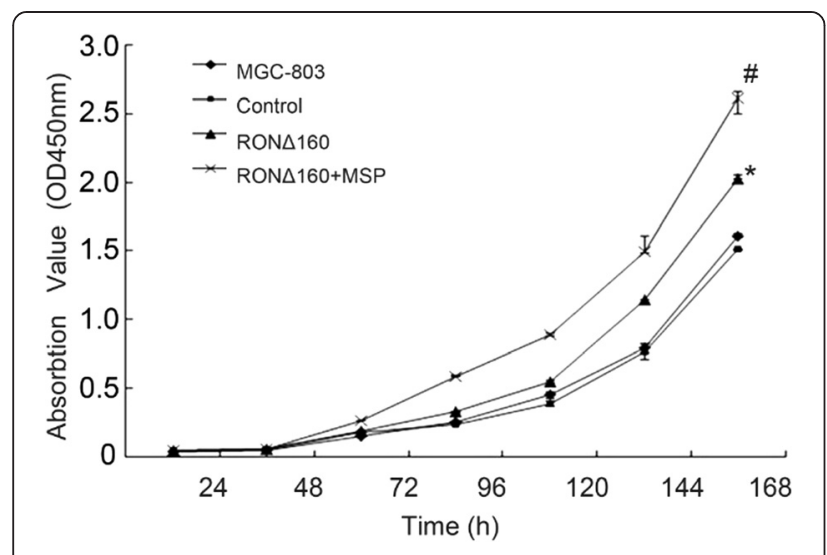

Figure 2 RON $\Delta 160$ improves the proliferation of MGC-803 cells, and MSP increases this effect. \#: $p<0.05$ (compared with the MGC-803 Group, empty vector control and RON 160 Group); *: $p<0.05$ (compared with the MGC-803 Group and empty vector control).

P-value less than 0.05 was considered to be statistically significant.

\section{Results}

Recombinant plasmid pcDNA3.1-RON $\Delta 160$ stably transfected into MGC-803 cells induces morphological changes

After screening MGC-803 cells for transfection with the recombinant plasmid pcDNA3.1-RON $\Delta 160$, western blotting and real-time quantitative PCR verified that
pcDNA3.1-RON $\Delta 160$ was successfully transfected into MGC-803 cells and expressed. The RON $\Delta 160$ protein and mRNA expression levels of the RON $\Delta 160$ group cells were significantly higher than those of the control and MGC-803 group ( $\mathrm{p}<0.01)$. The empty vector control and MGC-803 group showed almost no expression (Figure 1A,B). The cell morphology of the RON $\Delta 160$ group changed from the previous flat circular shape or polygon to a spindle shape (Figure $1 \mathrm{C}$ ).

\section{RON $\Delta 160$ improves the proliferation of MGC-803 cells}

Cell proliferation was measured with the CCK- 8 cell proliferation assay. The results showed that the overexpression of RON $\Delta 160$ promoted significant proliferation of MGC- 803 cells in the RON $\Delta 160$ group compared with the MGC-803 and empty vector control $(p<0.05)$. Meanwhile, the cell proliferation in cells overexpressing RON $\Delta 160$ and stimulated with MSP (the RON $\Delta 160+$ MSP group) was significantly higher than in the RON $\Delta 160$ group, MGC-803 group and empty vector control $(p<0.05)$ (Figure 2).

RON $\Delta 160$ increases the invasive capacity of MGC-803 cells Cell invasion capacity was reflected by the number of cells that penetrated the bottom chamber of the Transwell chamber through the artificial substrate Matrigel that simulates the cell outer basement membrane. The results showed that the invasive capacity of MGC-803
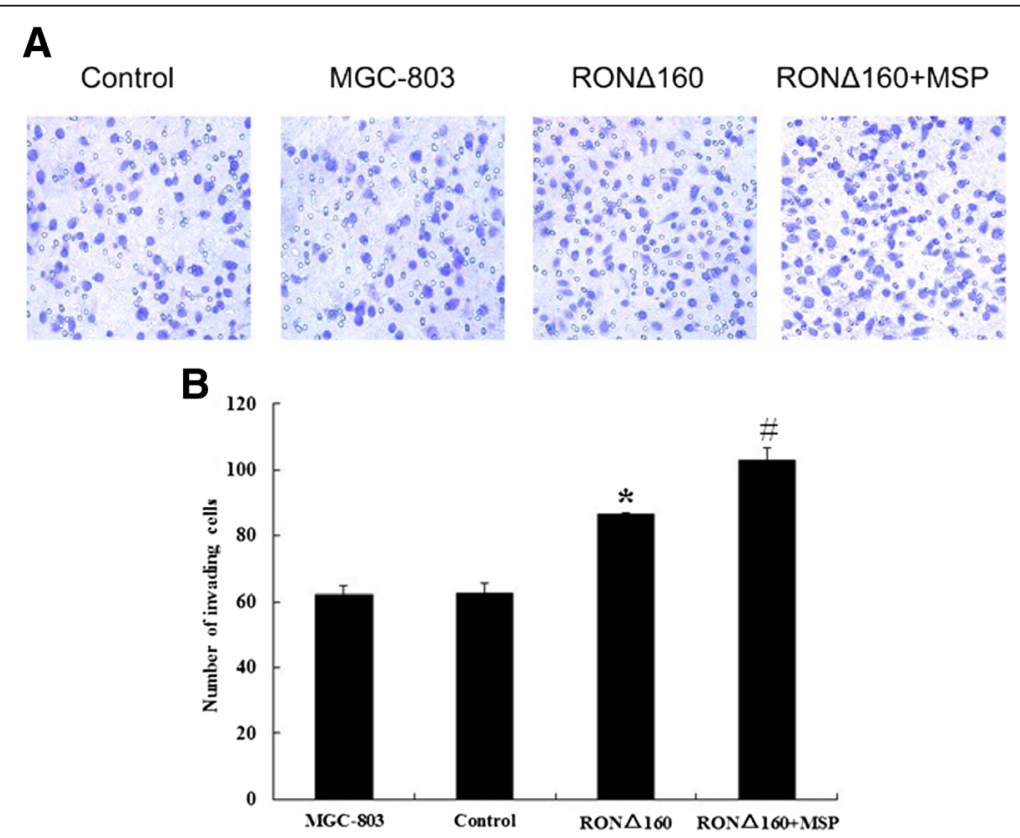

Figure 3 RON $\Delta 160$ increases the invasive capacity of the MGC-803 cell line ( $\times \mathbf{4 0 0})$. A: Compared with MGC-803 group (61.95 \pm 2.78$)$, empty vector control (62.35 \pm 3.32 ) and RON $\Delta 160$ group (86.48 \pm 0.32$), p<0.05$; compared with RON $\Delta 160$ group (86.48 \pm 0.32 ) and RON $160+$ MSP group (103.03 \pm 3.69$), p<0.05$; B: *: Compared with MGC-803 group, empty vector control, $p<0.05$; \#: Compared with MGC-803 group, empty vector control and RON $\Delta 160$ group, $p<0.05$. 


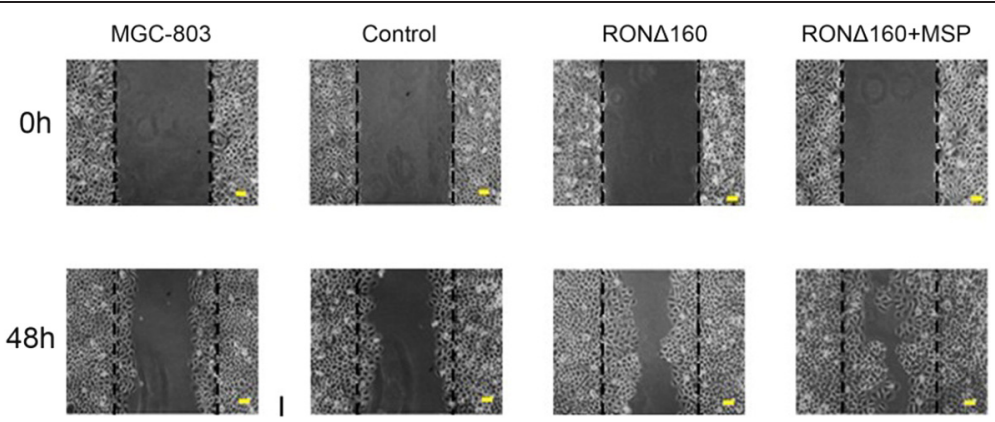

Figure 4 RON $\Delta 160$ increases the motility of MGC-803 cells $(\times 100)$. Compared with MGC-803 group, empty vector control and RON $\Delta 160$ group, $p<0.05$; Compared with MGC-803 group, empty vector control and RON $\Delta 160+$ MSP group, $p<0.05$; Compared with MGC-803 group and empty vector control, $p>0.05$.

cells of the RON $\Delta 160$ group $(86.48 \pm 0.32)$ was significantly higher than that of MGC-803 group (61.95 \pm 2.78$)$ and the empty vector control $(62.35 \pm 3.32)(p<0.05)$. Meanwhile, the cell invasive capacity of the RON $\Delta 160+$ MSP group $(103.03 \pm 3.69)$ was significantly higher than the RON $\Delta 160$ group $(p<0.05)$ (Figure $3 \mathrm{~A}, \mathrm{~B})$.

\section{RON $\Delta 160$ promotes the motility of MGC- 803 cells}

The results of the wound healing assay showed that 48 hours after scratching, the migration capacity of cells of the RON $\Delta 160$ group $(50.41 \pm 2.09 \%)$ was significantly higher than that of the MGC-803 group $(28.70 \pm 1.21 \%)$ and the empty vector control $(26.43 \pm 1.69 \%)(p<0.05)$. The cell migration capacity of the RON $\Delta 160+$ MSP group was significantly enhanced compared with the RON $\Delta 160(71.18 \pm 2.74 \%)(p<0.05)$ (Figure 4$)$.

\section{RON $\Delta 160$ regulates the cell cycle activities of MGC-803 cells} The results showed that the proportions of cells in each phase of the cell cycle in the MGC-803 group, empty vector control, RON $\Delta 160$ group and RON $\Delta 160+$ MSP group were as follows: G0-G1 phase (57.63 $\pm 0.50 \%)$,
$(56.65 \pm 0.22 \%), \quad(50.78 \pm 0.59 \%)$, and $(32.91 \pm 0.66 \%) ; \quad S$ phase $(34.77 \pm 0.61 \%), \quad(35.67 \pm 0.78 \%), \quad(40.33 \pm 0.25 \%)$, and (55.22 $\pm 0.59 \%)$; and $\mathrm{G} 2-\mathrm{M}$ phase (7.61 $\pm 0.73 \%)$, $(8.69 \pm 0.80 \%),(8.90 \pm 0.35 \%)$, and $(11.72 \pm 0.30 \%)$. Compared with the MGC-803 group and the empty vector control, the proportions of $S$ phase cells of RON $\Delta 160$ group and RON $\Delta 160+$ MSP group were larger, the proportions of cells in G0-G1 phase were relatively smaller, and the proportions of cells in G2-M phase were not significantly different. There were significantly more cells in S and G0G1 phases in the RON $\Delta 160+$ MSP group $(p<0.05)$. The proportions of cells in each phase between the MGC-803 group and the empty vector control were not significantly different $(p>0.05)$. The above results showed that, in terms of the cell cycle, overexpression of RON $\Delta 160$ promoted proliferation of MGC-803 cells (Figure 5).

\section{Overexpression of RON $\Delta 160$ promotes tumor growth} in vivo and its specific small molecule inhibitor can inhibit tumor growth

We further explored the influence of RON $\Delta 160$ on tumor growth of MGC-803 cells in tumor-bearing nude

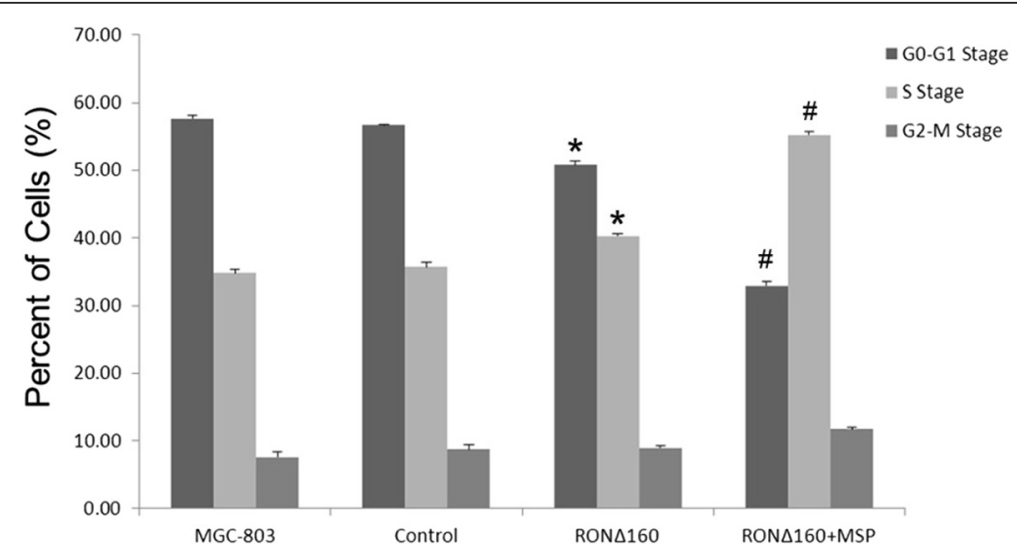

Figure 5 RON $\Delta 160$ results in an arrest in the $\mathbf{S}$ phase of the cell cycle in MGC-803 cells. *: $p<0.05$ (compared with the MGC-803 Group and empty vector control); \#: $p<0.05$ (compared with the MGC-803 Group, empty vector control and RON 160 Group). 
Table 1 The effects of RON $\Delta 160$ and c-Met/RON small cellular inhibitor on MGC-803 cells tumor-bearing nude mice

\begin{tabular}{lllll}
\hline & RON $\mathbf{\Delta 1 6 0}$ Group* & CP-I Group & MGC-803 Group & Empty vector control \\
\hline Tumor volume $\left(\mathrm{mm}^{3}\right)$ & $2703.79 \pm 92.66$ & $1743.77 \pm 119.75$ & $1176.36 \pm 149.13$ & $1273.17 \pm 316.23$ \\
Tumor weight $(\mathrm{g})$ & $1.93 \pm 0.11$ & $1.21 \pm 0.33$ & $0.89 \pm 0.29$ & $0.91 \pm 0.41$ \\
Tumor incubation period (days) & $12 \pm 1$ & $14.5 \pm 1.5$ & $20 \pm 1.5$ & $19.7 \pm 2.52$ \\
\hline
\end{tabular}

*: $p<0.05$ (compared with MGC-803 Group, empty vector control); $: p<0.05$ (compared with RON $\Delta 160$ Group).

mice. The results showed that compared with the MGC803 group and empty vector control, the tumorigenic ability of cells of the RON $\Delta 160$ group was significantly enhanced in vivo by promoting tumor growth, shortening the incubation period of tumor formation and increasing the volume and weight of the tumors. In contrast, compared with the RON $\Delta 160$ group, the CP-I group injected with small molecule inhibitor c-Met/ RON (Compound I) showed the inhibition of the tumorigenic superiority conferred by RON $\Delta 160$ in vivo $(\mathrm{p}<0.05)$ (Table 1, Figure 6).

\section{Discussion}

RON is a receptor with unique tyrosine kinase activity and is a member of the proto-oncogene c-Met family. RON may be activated by ligand-dependent or non ligand-dependent methods. Currently, MSP, which is synthesized by liver cells, is the only known specific ligand of RON. In our previous study, we showed that the expression ratio of RON in gastric cancer tissues was $56.1 \%$, and the corresponding expression ratio in adjacent tissues was $25.6 \%$, whereas there is no or little expression in the normal gastric mucosa. The expression level of RON is directly related to tumor invasion, lymph node metastasis and pathological (TNM) stages, which implies that RON plays an important role in tumorigenesis and development of gastric cancer [12]. Similarly, Song et al. found that the expression of $\mathrm{RON}$ in gastric cancer and lymph node metastasis tissue is higher than that in normal gastric mucosa and lymph node tissue without metastasis [13]. The study results of Catenacci et al. showed that RON is overexpressed in $74 \%$ of cancer specimens from the gastroesophageal junction; thus, they demonstrated that $\mathrm{RON}$ was an important prognostic factor and a potential biological therapeutic target [14]. Hence, RON plays an important role in the pathogenesis of gastric cancer.

Generally speaking, overexpression of RON is accompanied by the generation of variants. Most of the variants can promote the formation of tumors and increase invasion capacity. Studies have shown that the generation of variants plays an important role in maintaining diversified functions of proteins [15]. Some of the RON variants have antagonistic activity to wild-type RON. Other variants, for example RON $\Delta 170$, which lacks 46 amino acids encoded by the $19^{\text {th }}$ exon in the kinase domain, is unable to transmit a signal and inhibit formation of tumor [16]. However, most RON variants activate various cell signaling cascades through different substrate specificities and phosphorylation modes, thereby altering cell migration, invasion and proliferation, and promoting the formation of an invasive phenotype and tumor development, e.g. RON $\Delta 160[2,17]$. RON $\Delta 160$ is formed by a lack of exons 5 and 6, which encode 109 amino acids of the first IPT unit in the extracellular domain of the RON $\beta$ chain. RON $\Delta 160$ is constitutively activated by phosphorylation of residual groups of tyrosine and can withstand anti-RON antibody-mediated receptor internalization, which contributes to its maintenance of the in-cell signaling cascades and promotes the in vitro transformation of cells and the in vivo growth of tumors $[5,11]$.

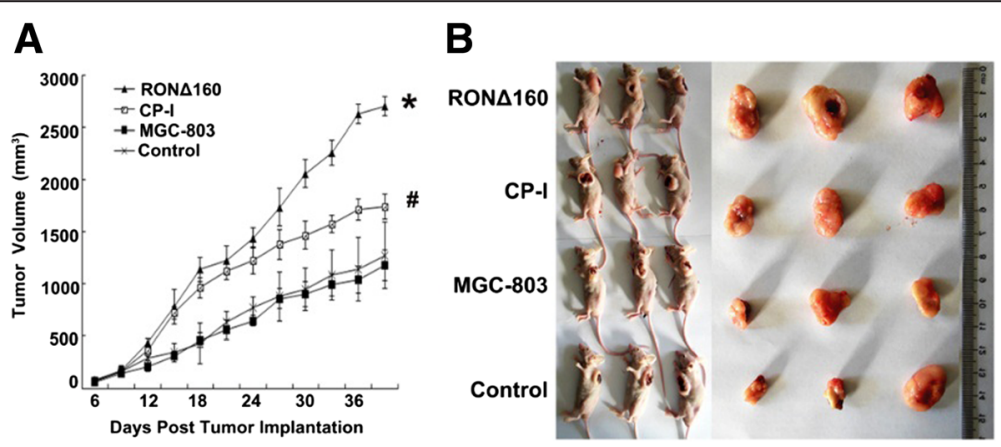

Figure 6 In vivo effects of RON $\mathbf{1 6 0}$ and its specific inhibitor Compound I on MGC-803 cells. A), *: $p<0.05$ (compared with the MGC-803 Group and empty vector control); \#: $p<0.05$ (compared with the RON 160 Group). B), after implanting tumor cells subcutaneously for 40 days, the nude mice were killed by cervical dislocation, and the tumors were peeled off and photographed. 
We found there was almost no expression of RON and its variant RON $\Delta 160$ in the human gastric cancer cell line MGC-803, which makes it a good in vitro carrier for studying the effect of RON on the bioactivity of gastric cancer cells. Through stable-transfection of MGC-803 cells with a variant RON $\Delta 160$ expressing plasmid, the overexpression of RON $\Delta 160$ in gastric cancer cells was successfully simulated. The observed change in the shape of RON $\Delta 160$ overexpressing MGC-803 cells from the previous flat circular shape or polygon to a spindle is similar to the result of $\mathrm{Xu}$ et al. [18], which confirmed that overexpression of RON changes the original shape of MDCK cells. The change of cell shape is likely related to RON $\Delta 160$-mediated epithelial-mesenchymal transition (EMT), i.e. losing the normal characteristics of epithelial cells and manifesting a malignant transformed mesenchymal cell phenotype [6]. In addition, the cell proliferation, migration and invasion assays showed that overexpression of RON $\Delta 160$ significantly promotes proliferation, migration and invasion of MGC-803 cells in vitro. These effects were significantly enhanced after stimulation with RON's sole specific ligand MSP, which further confirmed the tumorigenicity of variant RON $\Delta 160$. Meanwhile, cell cycle analysis also showed that RON $\Delta 160$ expressing cells are significantly more likely to be in the $\mathrm{S}$ phase and the proportion in the G0-G1 phase was relatively decreased, which further verified that the overexpression of RON $\triangle 160$ promotes proliferation of MGC-803 cells. To further explore the biological function of RON $\Delta 160$, the nude mice tumor-bearing animal model was used. The results showed that the in vivo tumorigenic ability of MGC803 cells stably transfected with the variant RON $\Delta 160$ plasmid is significantly increased via promotion of tumor growth, shortening of the incubation period of tumor formation and by increasing the volume and weight of the tumors. Addition of the small molecule inhibitor Compound I inhibited the tumorigenic effects of RON $\Delta 160$ and slowed the growth of the transplanted tumors in the nude mice. The RON/c-Met dual kinase inhibitor Compound I specifically interrupted the signal transduction pathways that are mediated by the hepatocyte growth factor and macrophage-stimulating protein, and reduces cell migration in a dosage-dependent way, both in vitro and in vivo [19]. The in vivo experiment conducted by Yao et al. showed that a RON monoclonal antibody could inhibit the activity of RON $\Delta 160$, which inhibits the growth of colon cancer cells and has a synergistic antitumor effect with cytotoxic drugs [20]. The above in vivo results further confirmed the tumorigenicity of RON $\triangle 160$ and indicate the possibility of using RON as the new therapeutic target for gastric cancer.

In summary, this is the first report of the influence of variant $\mathrm{RON} \Delta 160$ on the biological behavior of gastric cancer. The overexpression of variant RON $\Delta 160$ promotes the proliferation, migration and invasive capacity of a human gastric cancer cell line and its specific small molecule inhibitor can reverse the effects, possibly representing a novel route of targeted therapy. Therefore, our results provide a theoretical basis for the identifying new therapeutic targets for gastric cancer and the development of corresponding targeted drugs.

\section{Competing interests}

The authors declare that they have no competing interests.

\section{Authors' contributions}

$\mathrm{CL}$ and $\mathrm{LY}$ worked on oncobiological studies, animal studies, the analysis and interpretation of data; DZ designed the study and drafted the manuscript. All authors have reviewed the final version of the manuscript and approve it for publication.

\section{Acknowledgments}

This work was supported by grants from the National Natural Science Foundation of China (No.81272680) and the Natural Science Foundation of Zhejiang Province of China (No.Y2080168). We thank Hang-Ping Yao for his technical assistance.

\section{Author details}

'Department of Oncology, First Affiliated Hospital, Zhejiang University School of Medicine, Hangzhou 310003, China. 'Department of Oncology, Dongnan Affiliated Hospital of Xiamen University, Zhangzhou, China.

Received: 5 December 2013 Accepted: 5 January 2015

Published online: 04 February 2015

\section{References}

1. Leonis MA, Thobe MN, Waltz SE. Ron-receptor tyrosine kinase in tumorigenesis and metastasis. Future Oncol. 2007;3(4):441-8.

2. Xu XM, Zhou YQ, Wang MH. Mechanisms of cytoplasmic \{beta\}-catenin accumulation and its involvement in tumorigenic activities mediated by oncogenic splicing variant of the receptor originated from Nantes tyrosine kinase. J Biol Chem. 2005;280(26):25087-94.

3. Camp ER, Yang A, Gray MJ, Fan F, Hamilton SR, Evans DB, et al. Tyrosine kinase receptor RON in human pancreatic cancer: expression, function, and validation as a target. Cancer. 2007;109(6):1030-9.

4. Zhang K, Zhou YQ, Yao HP, Wang MH. Alterations in a defined extracellular region of the RON receptor tyrosine kinase promote RON-mediated motile and invasive phenotypes in epithelial cells. Int J Oncol. 2010;36(1):255-64.

5. Lu Y, Yao HP, Wang MH. Multiple variants of the RON receptor tyrosine kinase: biochemical properties, tumorigenic activities, and potential drug targets. Cancer Lett. 2007;257(2):157-64.

6. Ma Q, Zhang K, Guin S, Zhou YQ, Wang MH. Deletion or insertion in the first immunoglobulin-plexin-transcription (IPT) domain differentially regulates expression and tumorigenic activities of RON receptor Tyrosine Kinase. Mol Cancer. 2010;9:307.

7. Ma Q, Zhang K, Yao HP, Zhou YQ, Padhye S, Wang MH. Inhibition of MSP-RON signaling pathway in cancer cells by a novel soluble form of RON comprising the entire sema sequence. Int J Oncol. 2010;36(6):1551-61.

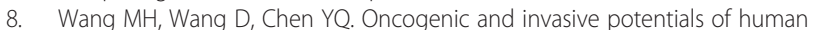
macrophage-stimulating protein receptor, the RON receptor tyrosine kinase. Carcinogenesis. 2003;24(8):1291-300.

9. Wang MH, Ronsin C, Gesnel MC, Coupey L, Skeel A, Leonard EJ, et al. Identification of the ron gene product as the receptor for the human macrophage stimulating protein. Science. 1994;266(5182):117-9.

10. Angeloni D, Duh FM, Moody M, Dean M, Zabarovsky ER, Sentchenko V, et al. C to A single nucleotide polymorphism in intron 18 of the human MST1R (RON) gene that maps at 3p21.3. Mol Cell Probes. 2003;17(2-3):55-7.

11. Yao HP, Luo YL, Feng L, Cheng LF, Lu Y, Li W, et al. Agonistic monoclonal antibodies potentiate tumorigenic and invasive activities of splicing variant of the RON receptor tyrosine kinase. Cancer Biol Ther. 2006;5(9):1179-86.

12. Zhou D, Pan G, Zheng C, Zheng J, Yian L, Teng X. Expression of the RON receptor tyrosine kinase and its association with gastric carcinoma versus normal gastric tissues. BMC Cancer. 2008;8:353. 
13. Song YA, Park YL, Kim KY, Myung E, Chung CY, Cho SB, et al. RON is associated with tumor progression via the inhibition of apoptosis and cell cycle arrest in human gastric cancer. Pathol Int. 2012;62(2):127-36.

14. Catenacci DV, Cervantes G, Yala S, Nelson EA, El-Hashani E, Kanteti R, et al. RON (MST1R) is a novel prognostic marker and therapeutic target for gastroesophageal adenocarcinoma. Cancer Biol Ther. 2011;12(1):9-46.

15. Pajares MJ, Ezponda T, Catena R, Calvo A, Pio R, Montuenga LM. Alternative splicing: an emerging topic in molecular and clinical oncology. Lancet Oncol. 2007:8(4):349-57.

16. Wang MH, Lao WF, Wang D, Luo YL, Yao HP. Blocking tumorigenic activities of colorectal cancer cells by a splicing RON receptor variant defective in the tyrosine kinase domain. Cancer Biol Ther. 2007;6(7):1121-9.

17. Santoro MM, Penengo L, Minetto M, Orecchia S, Cilli M, Gaudino G. Point mutations in the tyrosine kinase domain release the oncogenic and metastatic potential of the Ron receptor. Oncogene. 1998;17(6):741-9.

18. Xiangming X, Yun Q, Guoliang Z, Jianjiang L, Lisong T. Mechanisms of RON-mediated epithelial-mesenchymal transition in MDCK cells through the MAPK pathway. Braz J Med Biol Res. 2011;44(7):634-41.

19. Zhang Y, Kaplan-Lefko PJ, Rex K, Yang Y, Moriguchi J, Osgood T, et al. Identification of a novel recepteur d'origine nantais/c-met small-molecule kinase inhibitor with antitumor activity in vivo. Cancer Res. 2008;68(16):6680-7.

20. Yao HP, Zhou YQ, Ma Q, Guin S, Padhye SS, Zhang RW, et al. The monoclonal antibody Zt/f2 targeting RON receptor tyrosine kinase as potential therapeutics against tumor growth-mediated by colon cancer cells. Mol Cancer. 2011;10:82.

\section{Submit your next manuscript to BioMed Central and take full advantage of:}

- Convenient online submission

- Thorough peer review

- No space constraints or color figure charges

- Immediate publication on acceptance

- Inclusion in PubMed, CAS, Scopus and Google Scholar

- Research which is freely available for redistribution 